\title{
Oscillazioni libere del lago di Bracciano:
}

loro caratteristiche e loro energia $\left(^{*}\right)$

\author{
P. GALOI
}

Nelle due note precedenti $\left({ }^{1,2}\right)$, sono stati studiati, con metodi diversi, i possibili moti liberi del lago di Bracciano. I risultati sono riassunti nell'unita tabella: aver luogo, sebbene con maggior difficoltà di quelli asimmetrici.

La forma particolare del lago (non molto discosta da quella circolare), lasciava inoltre

Tabella 1

\begin{tabular}{|c|c|c|c|c|c|c|}
\hline \multirow{3}{*}{$\begin{array}{c}\text { Oscillazione } \\
\text { libera }\end{array}$} & \multicolumn{4}{|c|}{ Bacino circolare (Caloi) } & \multicolumn{2}{|c|}{ Canale a sez. variabile } \\
\hline & \multicolumn{2}{|c|}{ modo simmetrico } & \multicolumn{2}{|c|}{ modo asimmetrico } & \multirow[b]{2}{*}{ Caloi } & \multirow{2}{*}{$\begin{array}{l}\text { Metodo } \\
\text { Hidaka }\end{array}$} \\
\hline & $\begin{array}{c}\text { profondità } \\
\text { uniforme }\end{array}$ & $\begin{array}{c}\text { profondità } \\
\text { variabile }\end{array}$ & $\begin{array}{c}\text { profondità } \\
\text { uniforme }\end{array}$ & $\begin{array}{c}\text { profondità } \\
\text { variabile }\end{array}$ & & \\
\hline & m & $\mathrm{m}$ & $\mathrm{m}$ & $\mathrm{m}$ & $\mathrm{m}$ & $\mathrm{m}$ \\
\hline $\mathrm{T}_{0}$ & 4,0 & 4,0 & 8,4 & 8,0 & 8,2 & 8,8 \\
\hline $\mathrm{T}_{1}$ & 2,2 & 2,3 & 2,9 & 3,0 & 5,1 & 6,0 \\
\hline $\mathrm{T}_{2}$ & 1,5 & 1,6 & 1,8 & 1,9 & 3,5 & 3,7 \\
\hline
\end{tabular}

\section{STAZIONI D'OSSERVAZIONE.}

Nella fig. 1, riproducente il lago di Bracciano, sono segnate le stazioni che hanno funzionato dal 1947 al 1957, in modo intermittente. Alcune di esse hanno funzionato contemporaneamente: così, nel 1956, Valle dell'Inferno, La Sabatina, Bracciano I (e quindi Bracciano II) e Trevignano.

Come è stato provato in una nota precedente $\left(^{(1)}\right.$, i possibili modi d'oscillazione libera del lago di Bracciano - attesa la sua forma - non si esauriscono in quelli asimmetrici: anche i modi simmetrici possono

${ }^{*}$ ) Questo lavoro è stato condotto con contributi del Consiglio Nazionale delle Ricerche. prevedere la possibilità che i modi asimmetrici non si verificassero esclusivamente lungo un asse privilegiato. Di qui l'utilità della contemporanea registrazione agli estremi di due diametri incrociantisi, secondo un angolo non troppo piccolo.

\section{LIMNOGRAFI.}

Furono messi in funzione parecchi tipi di limnografi, alcuni dei quali di nuova concezione.

Uno di essi ha già fatto oggetto di una pubblicazione ${ }^{3}$ ).

Accennerò qui al tipo di apparecchio che più si è dimostrato idoneo alle osservazioni sistematiche. La fig. 2 ne dà una visione complessiva. 


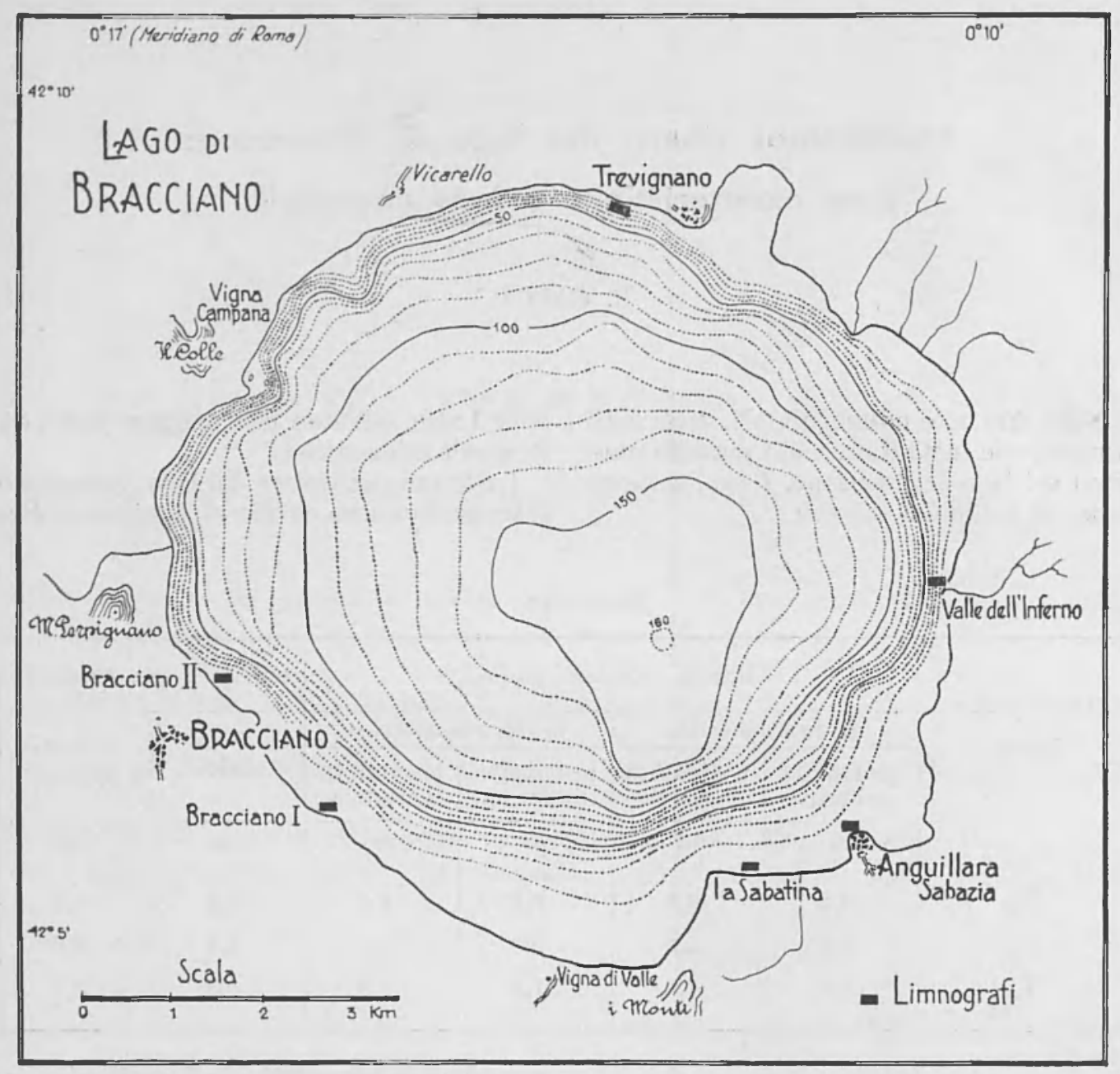

Fig. 1 - Stazioni limnografiche sul lago di Bracciano.

Un tubo di ottone, del diametro interno di circa $7 \mathrm{~cm}$, con la parte estrema mobile, che consente di allungare od accorciare il tubo stesso, quando viene infisso in riva al lago, in uno strato d'acqua conveniente. Nell'interno, il tubo è limitato da un cilindro di vetro, entro il quale si eleva l'acqua in cui è immerso un piccolo galleggiante, del diametro di $5 \mathrm{~cm}$, portante alle estremità di due diametri ortogonali, quattro rotelline girevoli intorno a piccoli assi orizzontali: in tal modo, il galleggiante può seguire i movimenti verticali dell'acqua praticamente senza attrito (Fig. 3). Nella sua parte superiore l'asticina del galleggiante (a sua volta variabile in lunghezza), è collegata, mediante una leggera molla piana, ad una leva girevole intorno ad un asse orizzontale. All'estremità libera, la leva porta una pennina con serbatoio per l'inchiostro. Poggiando sul registratore, la pennina scrive i movimenti del galleggiante, più o meno ingranditi. Lo scorrimento del registratore, viene regolato sui periodi delle sesse predominanti nei laghi allo studio.

Il congegno che rivela i moti dell'acqua, assieme all'apparato registratore, sono custoditi dentro ad una robusta scatola - che 


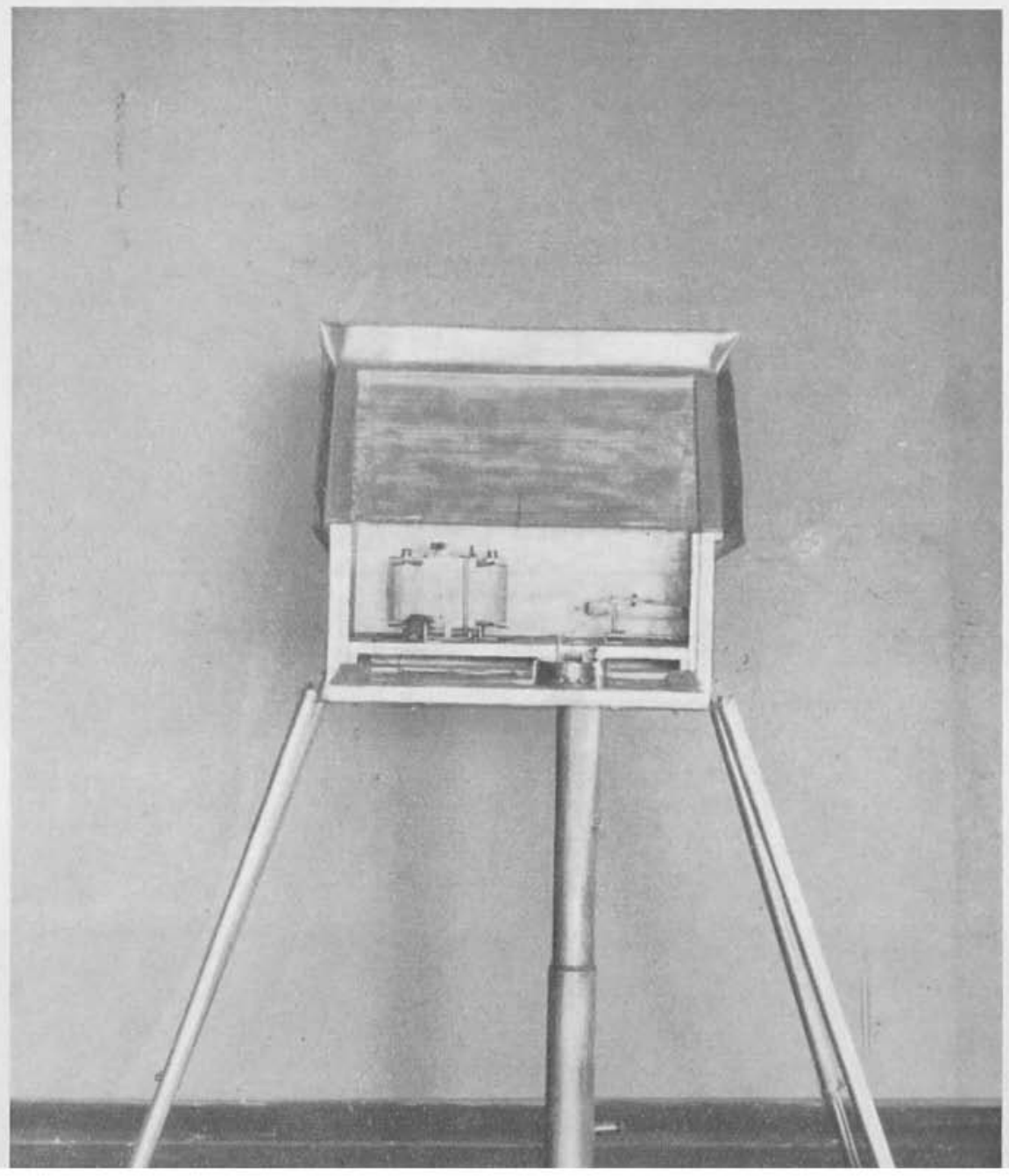




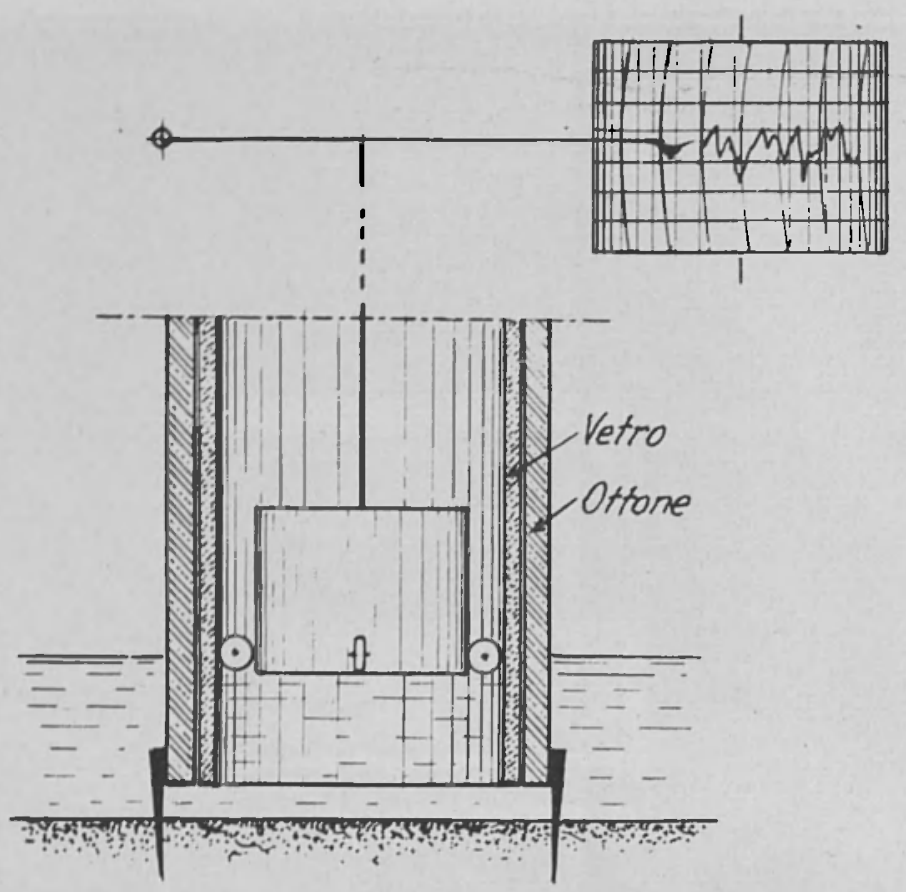

Fig. 3 - Particolari costruttivi del nuovo limnografo.

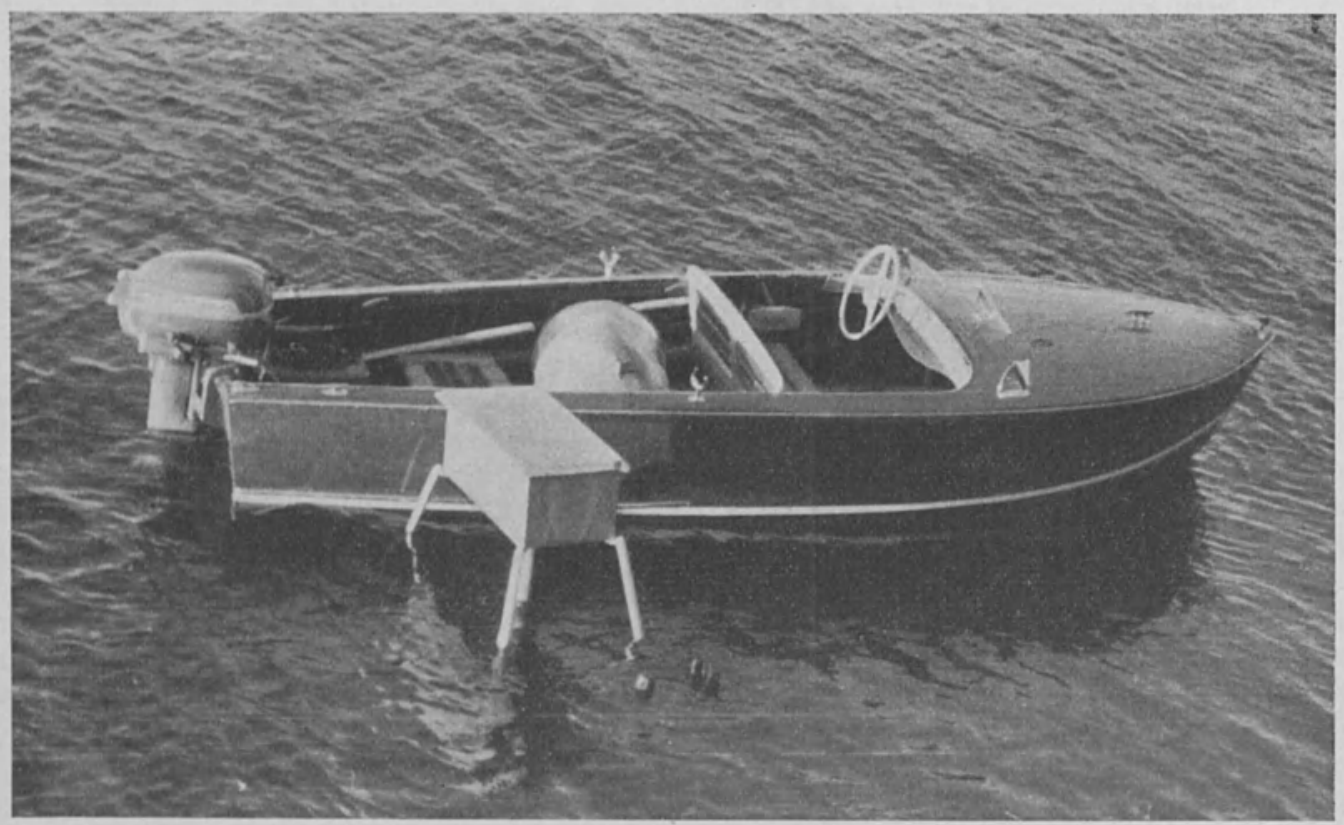

Fig. 4 - Limnografo in funzione sul lago di Bracciano. 


\section{Valle dell'Inferno}
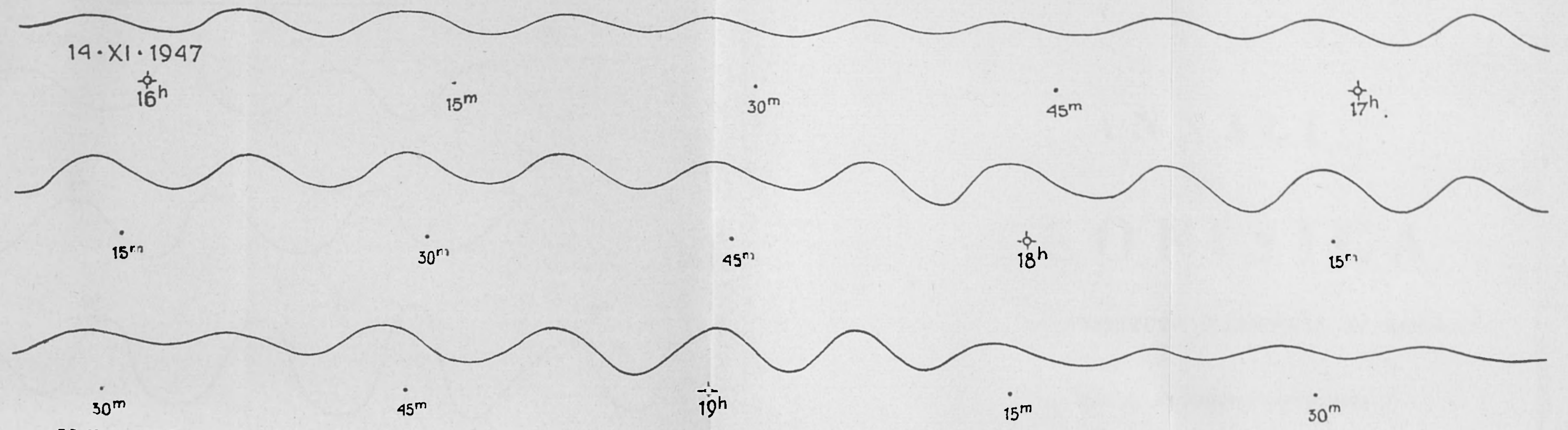

\section{Valle dell'Inferno}
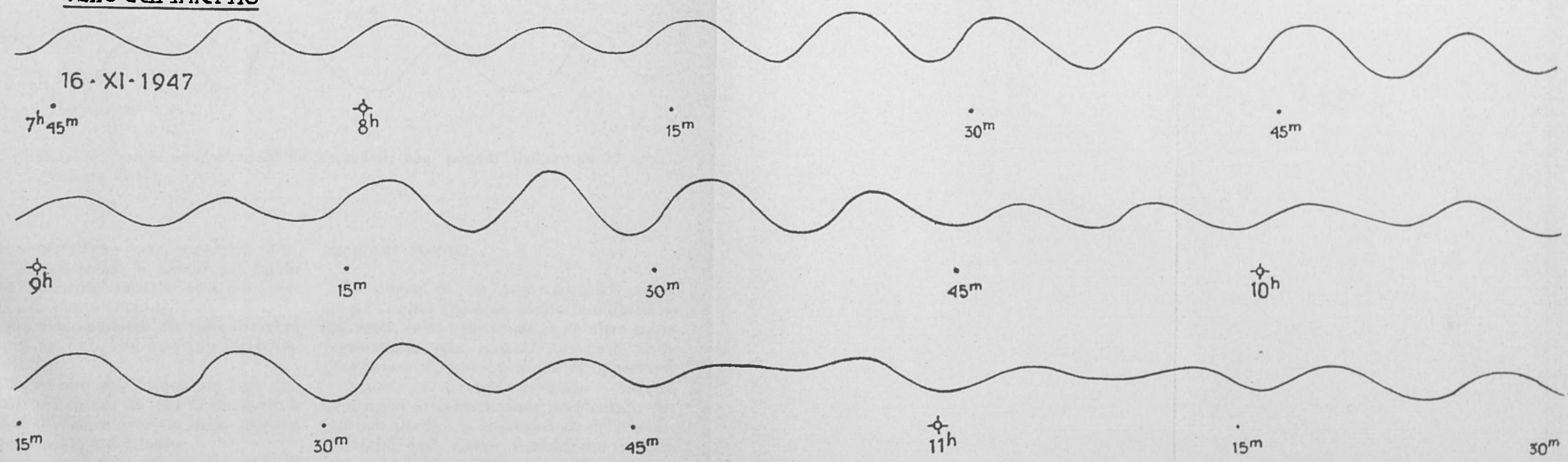

Fig. 5 - Lunga serie di oscillazioni libere uninodali quasi pure $(T=8,0 \div 8,2$ minuti $)$. 


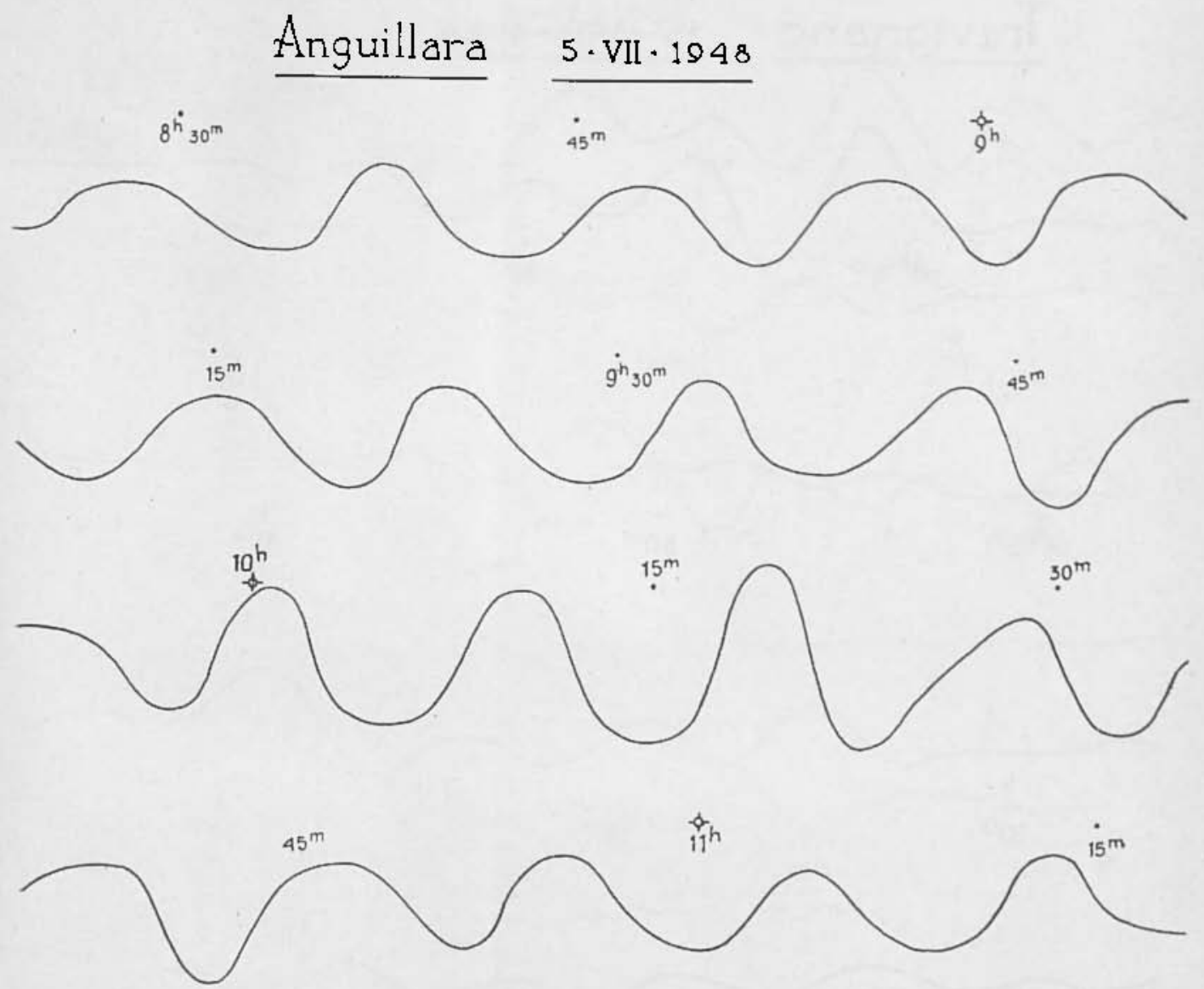

Fir. 6 - Esempi di ampie oscillazioni libere uninodali, con periodi dell'ordine di 8m,3. (Rirlotte $(3 \mathrm{i} 1 / 3$ ).

può essere mefallica - con coperchio sollevabile. L'apparecchio è fissato sul fondo sabbioso mediante quattro sostegni, terminanti con punte (Fig. 4).

L'acqua viene addotta nel tubo di vetro attraverso un foro, con apertura regolabile a piacere.

Il collegamento con l'aequa del lago può realizzarsi per mezzo di tubi di diametro e lunghezza diversi, a seconda dello scopo a cui l'apparecchio è destinato.

Le figure da 5 a 11 danno una serie di esempi di oscillazioni libere, registrate (escluse quelle che si riferiscono alle figg. 5 e 6 ) con limnografi del tipo descritto.

\section{RICHIAMI TEORICI.}

Le acque di un lago possono essere mosse - oltre che dalle oscillazioni libere dai venti, testi o fluttuanti, o da altre cause perturbanti, che possono generare onde progressive di breve periodo. E opportuno - quando ciò si rende necessario - ridurre al minimo le perturbazioni accidentah, che possono alterare la registrazione delle sesse. Talvolta, può essere vantaggioso ridurre altresi le oscillazioni libere multinodali, allo scopo di ottenere le sesse di più piecola nodalità il più possibile pure.

$\mathrm{Si}$ può raggiungere l'effetto desiderato 


\section{Trevignano $\quad \underline{15 \cdot v m \cdot 1956}$}
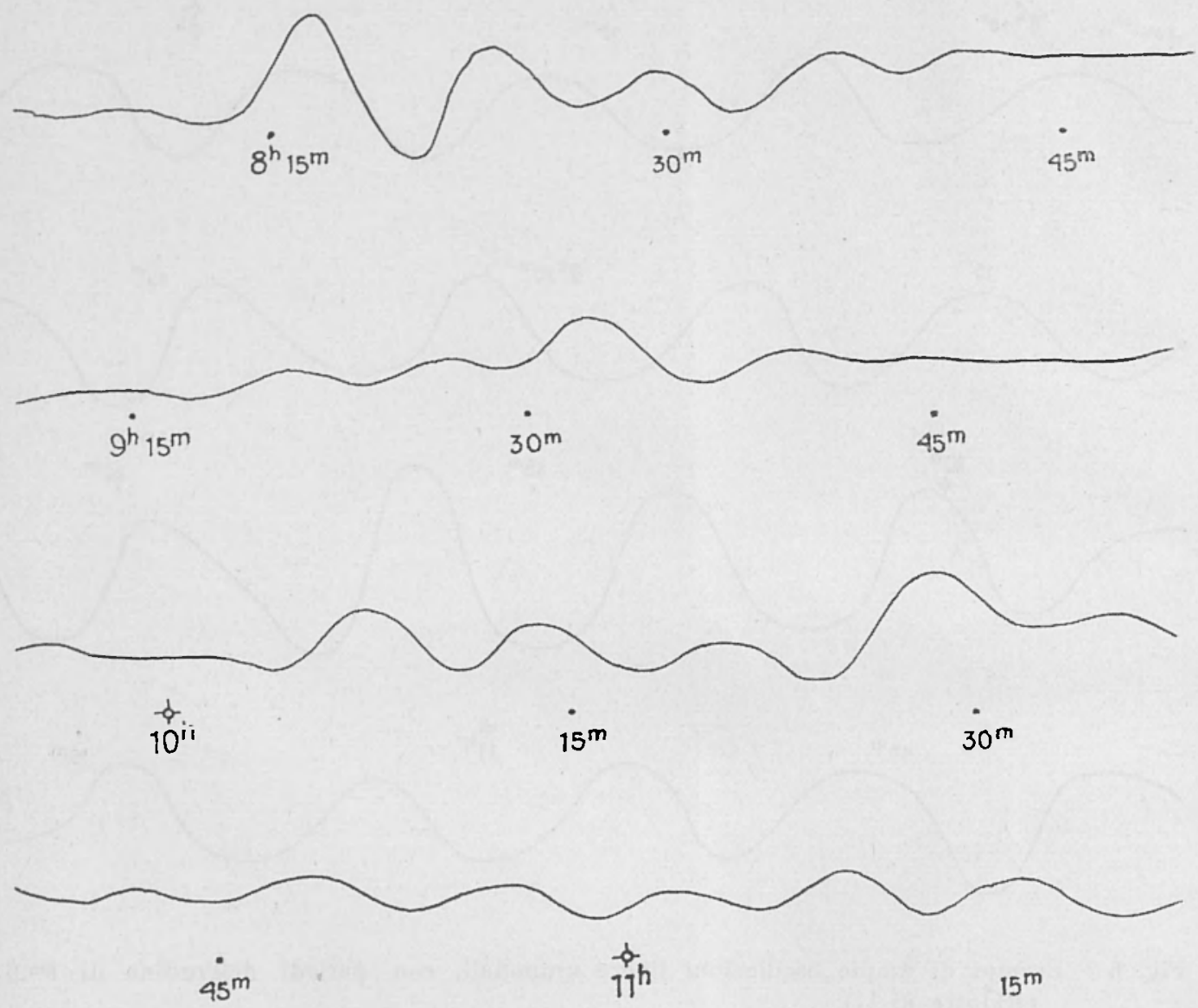

Fig. 7 - Esempi di sesse "dicrote ».

facendo sufficientemente piccolo il rapporto fra il diametro del tubo d'accesso e quello del pozzetto del galleggiante. Infatti com'è evidente - l'assottigliamento o l'allungamento del tubo d'accesso diminuisce l'ampiezza, nel limnogramma, dei disturbi di breve durata o di breve periodo. Nello stesso tempo, determina un ritardo nei massimi e nei minimi registrati, sui massimi e i minimi dei dislivelli effettivi del lago. In tal modo, restringendo od allungando il tubo d'accesso - o praticando entrambi gli accorgimenti -, non solo eliminiamo $\mathbf{i}$ disturbi dovuti al vento 0 al moto ondoso, ma riduciamo altresì le ampiezze delle sesse di più alta nodalità.

Calcoliamo l'effetto di smorzamento e di ritardo del tubo adduttore e del cilindro del galleggiante.

Siano $a$ e $b$ i diametri del pozzo cilindrico e del tubo d'accesso rispettivamente; $l$ la lunghezza del tubo; $x$ ed $y$ le quote del livello dell'acqua nel cilindro e nel lago, sopra un punto determinato, cioè l'estremità esterna del tubo d'accesso.

Il fisico inglese Osborne Reynolds $\left({ }^{4}\right)$ che fissò le leggi fondamentali della turbolenza - basandosi su numerosi esperimenti 

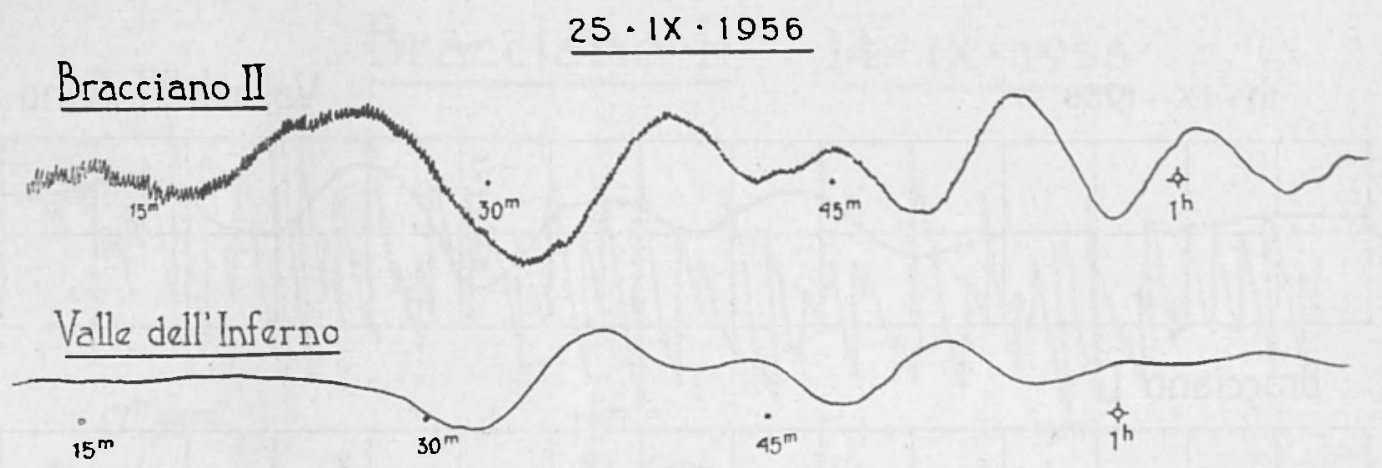

\section{Bracciano II}

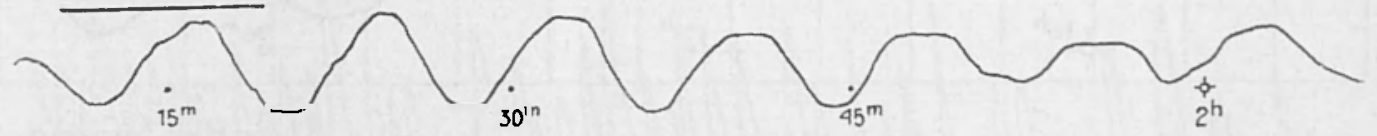

\section{Valle dell'Inferno}

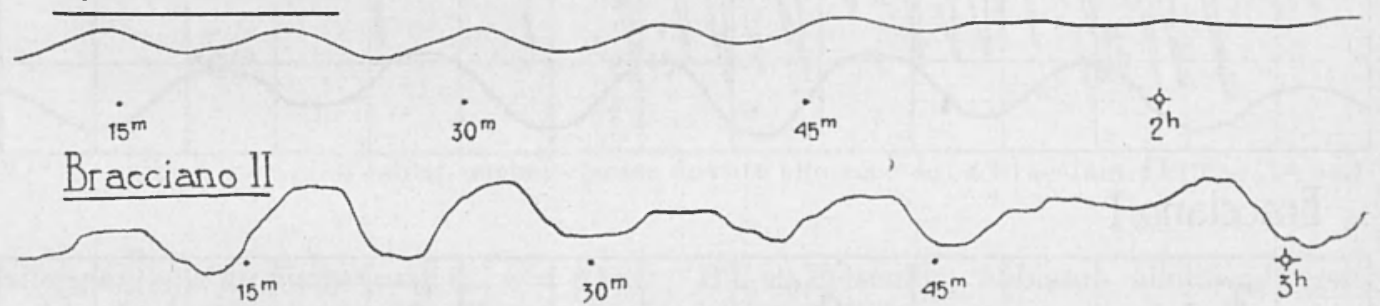

\section{Valle dell'Inferno}

$15^{m}$

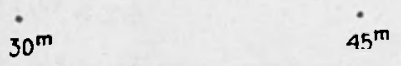

点

Fig. 8 - Oscillazioni varie per micropulsazioni della pressione atmosferica. (Ridotte di circa $1 / 3$ ).

compiuti con vari liquidi in tubi di diverso diametro, dimostró che la velocità critica per la formazione della turbolenza varia in proporzione diretta alla viscosità del liquido ed in proporzione inversa al diametro del tubo.

In particolare, nel caso dell'acqua, se la velocità nel tubo adduttore non eccede $V=2008-\frac{\nu}{b}$ - dove $\nu$ e la viscosità dell'acqua alla temperatura $\vartheta \circ \mathrm{C}$ e vale $\nu=.0178 /\left(1+.0337 \vartheta+.000221 \vartheta^{2}\right)-$, allora la resistenza nel tubo sarà proporzionale alla velocità media del flusso; in tal caso, è applicabile la formula di Poiseuille per calcolare la differenza di pressione alle due estremità del tubo per una data velocità del flusso (regime di Poiseuille).

Per una sessa di ampiezza registrata $B$, e di periodo $T$, la massima velocità di ascesa o di discesa del livello è, evidentemente, $\frac{2 \pi}{T} \cdot B$, che provoca nel tubo d'accesso la velocità $v=\frac{2 \pi}{\bar{I}^{i}} B \frac{a^{2}}{b^{z}}$. Per es., nel lago di Bracciano, la sessa uninodale eccede raramente l'ampiezza totale di $4 \mathrm{~cm}(B=2)$ Nel limnografo qui cennato, il pozzetto del 


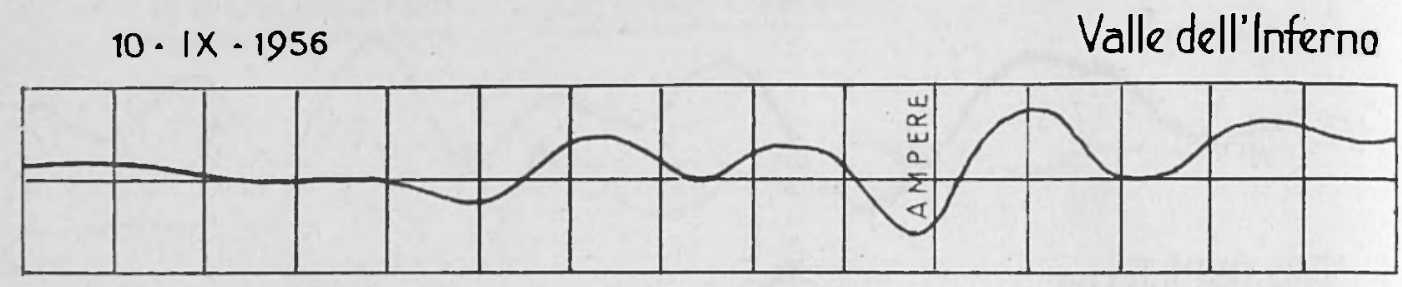

\section{Bracciano I}

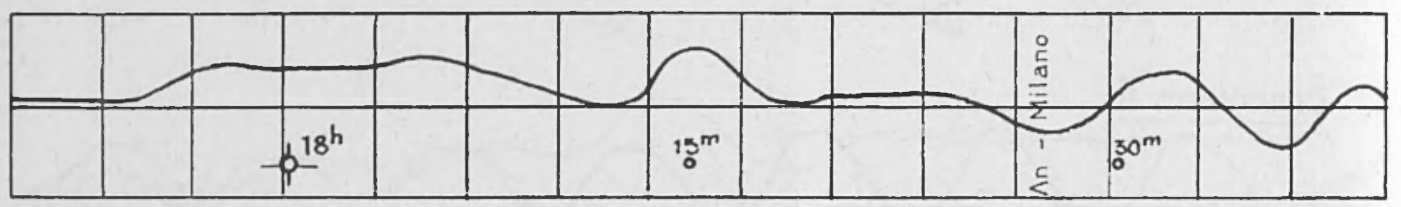

Valle dell'Inferno

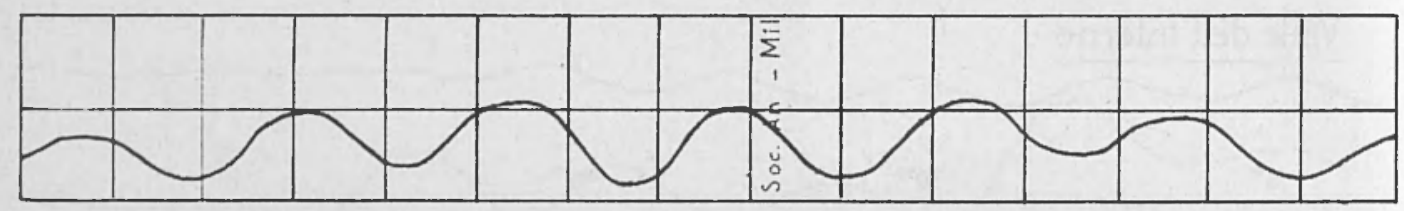

\section{Bracciano I}

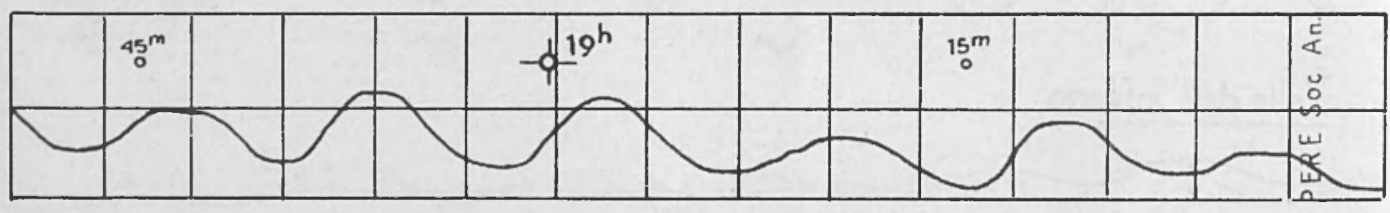

Valle dell'Inferno

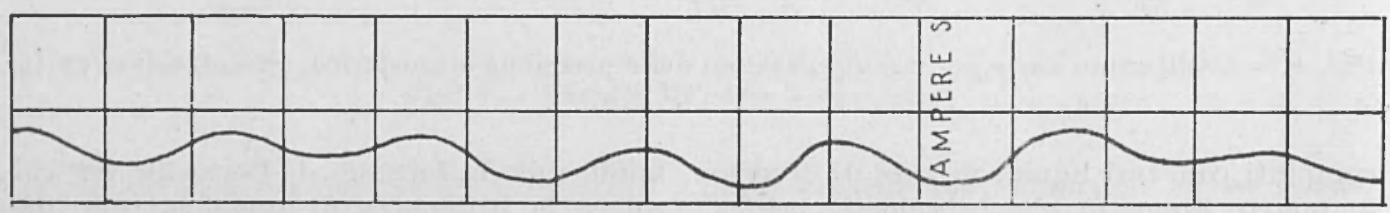

Bracciano I

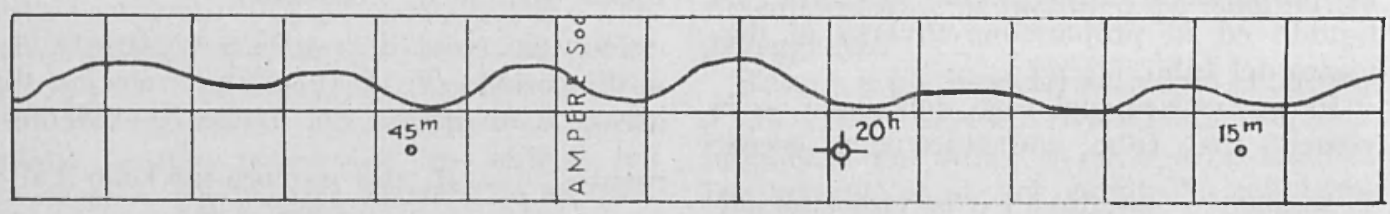

Fig. 9 - Perfetta opposizione Valle dell'Inferno - Bracciano I (uninodale). 


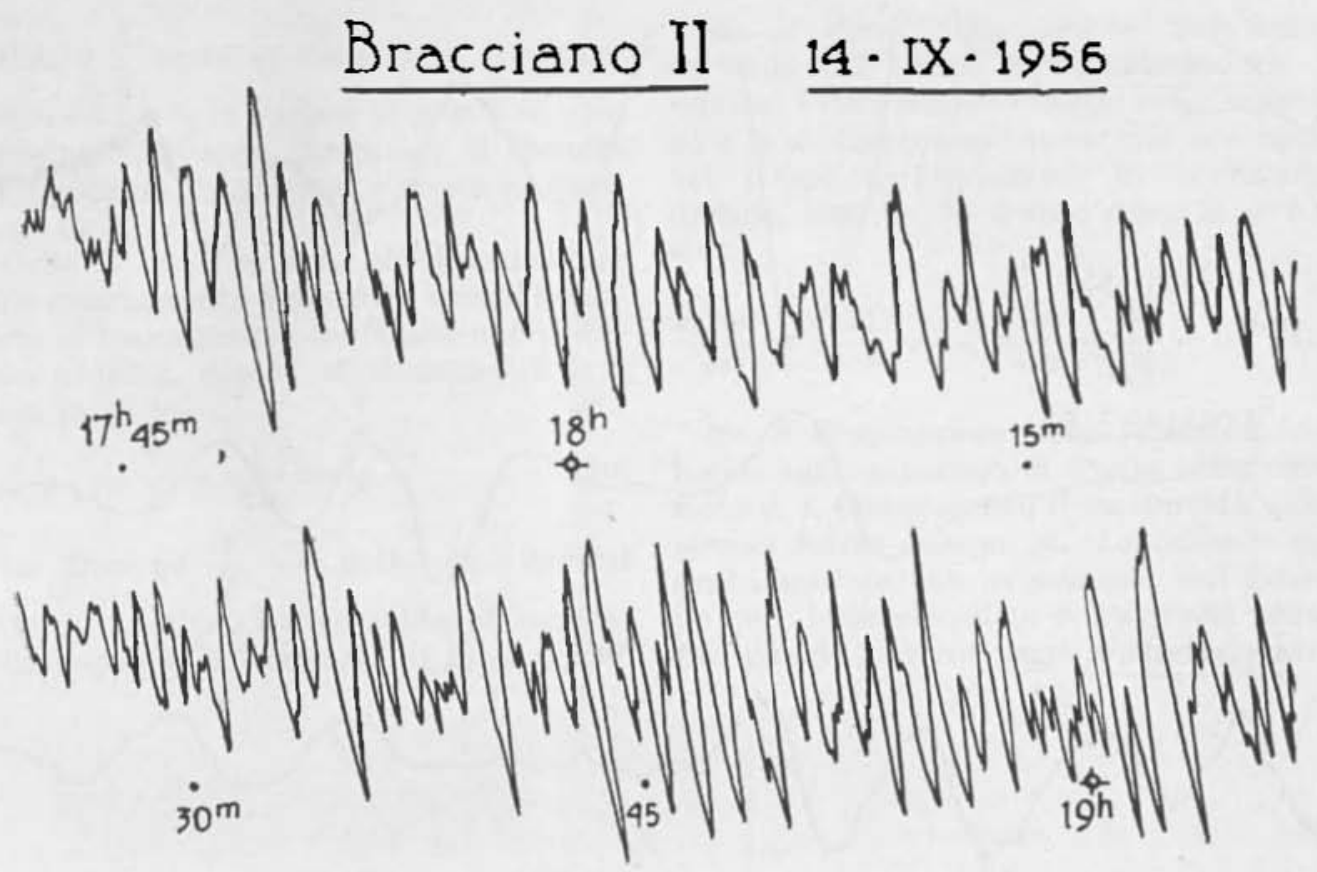

Fig. 10 - Chari esempi di «shelf-seishes" (sesse dovute allo zoccolo) a Braceiano II $(T=$ w ca.)

galleggiante ha un diametro di ca. $a=6 \mathrm{~cm}$. Prendendo la temperatura dell'acqua pari a. $\vartheta=18{ }^{\circ} \mathrm{C}$, cosi che $v=.010607$, otteniamo per la sessa uninodale del lago di Bracciano $\left(T_{\mathrm{o}}=500_{\mathrm{s}}^{\mathrm{s} \text { ca. })}\right.$

$$
\nabla=21,30 / b \quad, \quad v=.9043 / b^{2} \text { c.g.s. }
$$

A seconda dei diametri del tubo di accesso, avremo quindi per $V . v$ rispettivamente

\begin{tabular}{|c|c|c|}
\hline$b$ & $Y$ & $v$ \\
\hline \multirow{2}{*}{$1.0 \mathrm{~cm}$} & $2 \mathrm{I}, 3$ & - \\
0.5 & 42,6 & 0.9043 \\
0.3 & 71,0 & 3,62 \\
0.2 & 106,5 & 22,6 \\
\hline
\end{tabular}

Il massimo attuale (v) è quindi, in ogni caso, ben al di sotto della velocità critica.

Poicho il moto è molto lento, può essere ritenuto come stazionario. Per la legge di
Hagen-Poiseuille, abbiamo allora ad ogni istante $t\left(^{5}\right)$.

$$
-\pi \frac{a^{2}}{4} \cdot \frac{d x}{d t}=\frac{\pi b^{4} g(x-y)}{128 v l} .
$$

Perciò, facendo

$$
\nu=0.010607, g=981 ;
$$

e ponendo:

$$
k=q \cdot b^{4} / 32 \cdot v \cdot a^{2} l=2890 \cdot \frac{b^{4}}{-},
$$

si ottiene

Sia il livello del lago soggetto a moto armonico di ampiezza $A$ e periodo $T$.

Se $\omega=\frac{2 \pi}{-}$ e $q$ è la profondità media all'estremità del tubo di accesso, avremo:

$$
y=A \sin \omega t+q .
$$

Ne segue

$$
+k x=k A \sin \omega t+k q .
$$



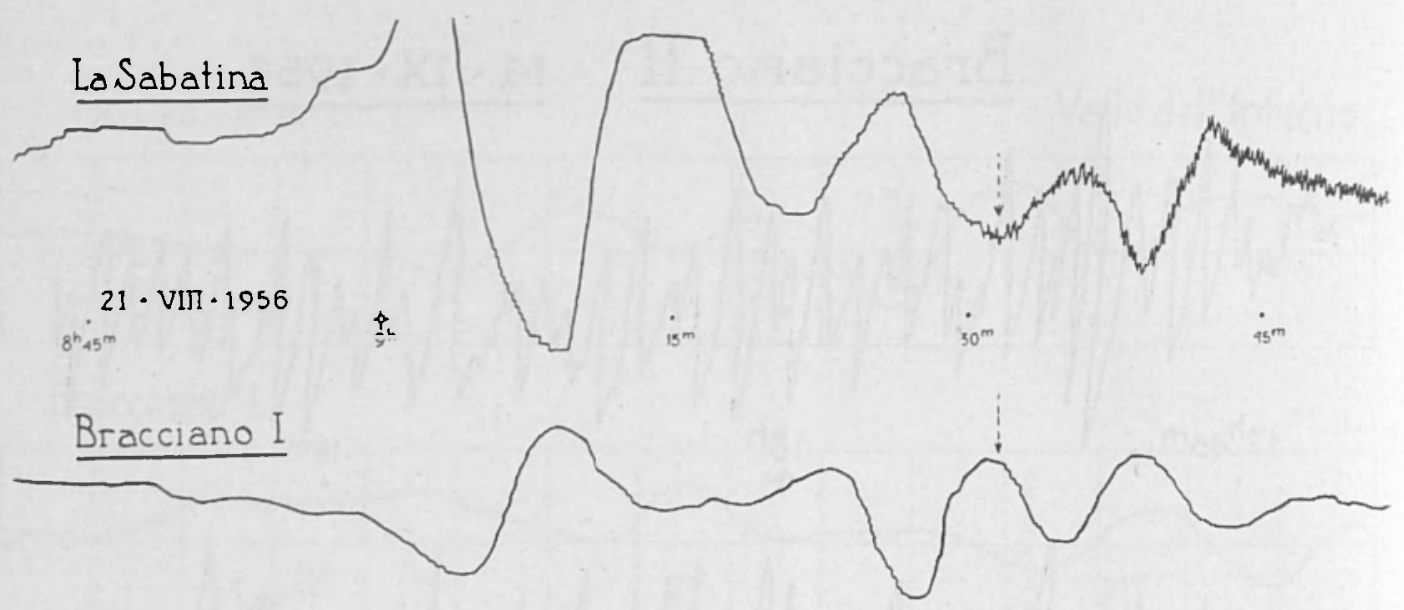

La Sabatina
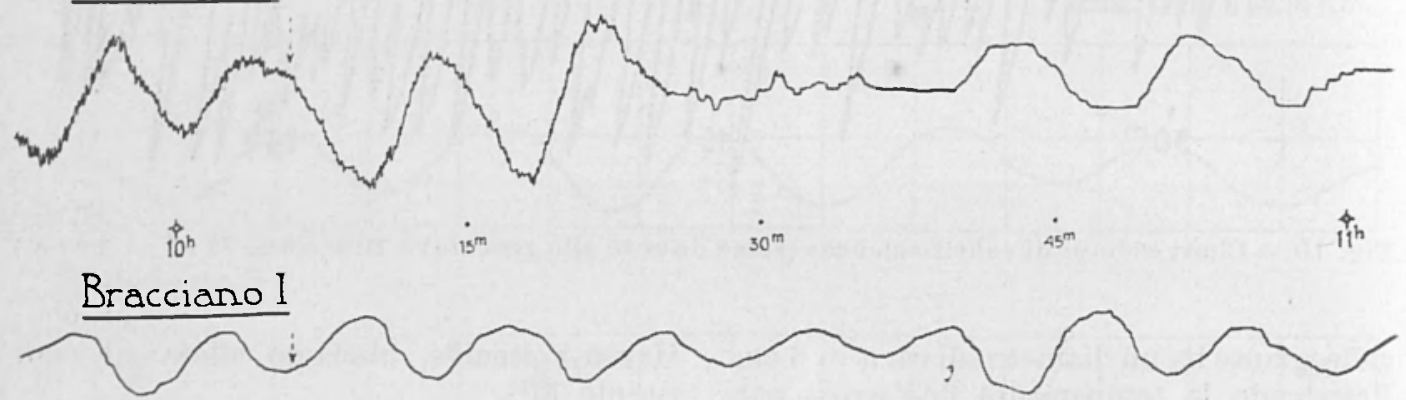

Fig. 11 - Opposizioni Bracciano II - Valle dell'Inferno, con progressiva variazione direzione piano diametrale d'oscillazione (riduzione di $1 / 3$ ca.).

La [4] è un'equazione differenziale lineare del primo ordine, il cui integrale è $x=e^{-k t}\left\{\int k A \sin \omega t e^{k t} d t+\int k q e^{k t} d t+C\right\}$.

Da cui

$x=q+C e^{-k t}-k A(\omega \cos \omega t-k \sin \omega t) /$ $/\left(\omega^{2}+k^{2}\right)$.

Posto

consegue

$$
\operatorname{tang} \omega \tau=\frac{\omega}{k}
$$

$x=q+C e^{-k t}+A \cos \omega \tau \sin \omega \cdot(t-\tau) .[6]$

Trascorso un certo tempo dall'inizio delle osservazioni, quando il livello dell'acqua nel pozzetto ha perduto le perturbazioni ini- ziali, il termine $C e^{-k t}$ può essere trascurato. Si ha allora

$$
x-q=A \cos \omega \tau \sin \omega(t-\tau) .
$$

L'oscillazione armonica del livello del lago, come è registrata dal limnografo, è ritardata dell'intervallo di tempo,

$$
\tau-\frac{1}{\omega} \operatorname{arctg} \frac{\omega}{k}
$$

e smorzata nel rapporto:

Fatto

$$
\cos \omega \tau: 1 \text {. }
$$

$$
\varphi=\frac{\omega}{k},
$$

avremo

$$
\tau=\frac{1}{k} \frac{\operatorname{arctg} \varphi}{\varphi} .
$$


Poichè $\frac{1}{\varphi} \operatorname{arctg} \varphi$ diminuisce continuamente da 1 a 0 , al variare di $\varphi$ da 0 a $+\infty$, segue che il ritardo diminuisce al crescere della nodalità della sessa, e cresce al diminuire di $k$.

Dalle [5] e [8] si nota che l'ammontare dello smorzamento aumenta e quindi il rapporto di smorzamento diminuisee al crescere della nodahtà, oppure al diminuire di $k$. Dalla [8] si ha

$$
\frac{\tau}{T}=\frac{1}{2 \pi} \operatorname{arctg} \frac{\omega}{7} \text {. }
$$

La frazione $-\frac{\tau}{T}$ - detta da Chrystal "ritardo relativo» - aumenta al crescere della nodalità, col crescere di $k$; e poichè
Sia $s$ l'area della sezione trasversale verticale nel punto $x$ considerato. Se $\zeta$ esprime l'innalzamento medio nella sezione ed $u$ la media corrente attraverso la sezione nel tempo $t$, l'equazione di continuità diviene, essendo, in questo caso, $S=b h$ cost.,

$$
\frac{\partial(S u)}{\partial x}+h \frac{\partial \zeta}{\partial t}=0, h \frac{\partial u}{\partial x}+\frac{\partial \zeta}{\partial t}=0 .
$$

Sia $\vartheta$ l'inclinazione della superficie isobariea sulla superficie di livello nella posizione $x, t$. Consideriamo il movimento della piccola massa d'acqua $m$. La corrente essendo sensibilmente orizzontale, tah saranno pure le accelerazioni relative alla terra. Per piccole correnti, come viene supposto,
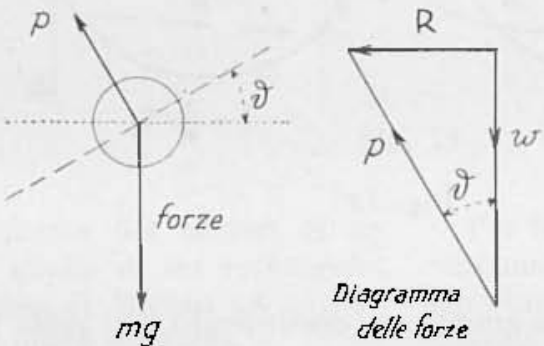

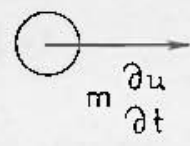

Accelerazione

dello massa $m$

Fig. 12 - Diagramma delle forze (secondo Proudman).

il massimo di aretg è $\frac{\pi}{2}$. il massimo possibile valore del ritardo relativo sarà $\frac{1}{4}$. Così il massimo possibile ritardo per una sessa è un quarto del suo periodo.

Le relazioni riportate ci serviranno specialmente neila registrazione delle onde interne (sesse termiche), su cui ci intratterremo in un prossimo lavoro.

VALORE APPROSSLMATIVO DELL'ENERGIA ASSOCLATA ALLE SESSE DEL LAGO DI BRACCIANO.

Abbiamo visto clie la forma del lago di Bracciano può pure essere assimilata a quella di un rettangolo. Tale riduzione conduce a valori per le oscillazioni libere molto prossimi a quelli osservati.

Sia $h$ la profondità media del bacino rettangolare di larghezza $b$. l'accelerazione è data da $\frac{\partial u}{\partial t}$ nella direzione delle $x$ crescenti. Sulla piccola massa d'acqua agiscono la forza peso $w$ verticalmente verso il basso e la pressione risultante $p$ perpendicolarmente alla superficie isobarica. La risultante di queste forze dev'essere nella direzione dell'accelerazione e quindi orizzontale (fig. 12).

Dal diagramma delle forze consegue

$$
R-w \operatorname{tang} \vartheta=m g \operatorname{tang} \vartheta
$$

nel senso delle $x$ decrescenti, e perciò

$$
m-=-m g \operatorname{tang} \vartheta .
$$

Eे d'altronde $\operatorname{tang} \vartheta \quad \frac{\partial \zeta}{\partial x}$. Pertanto

$\partial u$

$$
g_{\partial x}
$$


Consideriamo un bacino di forma allungata, in cui l'acqua si muova ritmicamente con periodo $T_{0}$. In una sezione verticale del lago, possono considerarsi quattro fasi di innalzamento e di correnti, dal tempo $t=o$ al tempo $t=T_{o}$ (fig. 13).

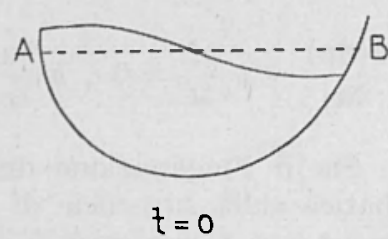

che. Dalla fig. 14 si trae

$$
u=C \sin \frac{\pi x}{\imath} \sin \frac{2 \pi}{I_{o}^{\prime}} t,
$$

dove $C$ è una velocità costante. La [13] soddisfa alle condizioni richieste.
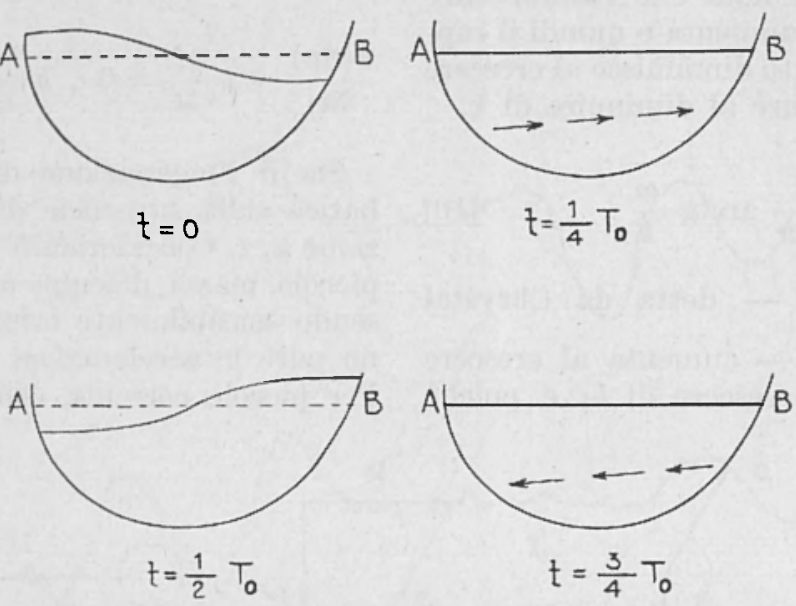

Fig. 13

In un periodo completo si hanno quindi quattro momenti, il $1^{\circ}$ e il $3^{\circ}(t=0$, $\left.t=\frac{1}{2} T_{0}\right)$ caratterizzati da massimi spostamenti verticali agli estremi $A, B$, di segno contrario nei due casi a ciascun estremo; il $2^{\circ}$ e il $4^{\circ}\left(t=\frac{1}{4} T_{o}, t=\frac{3}{4} T_{\mathrm{o}}\right)$, contraddistinti da superficie del lago livellata e dal massimo valore per le correnti, di segno nvertito nei due casi. Supposta uniforme la densità dell'acqua, il gradiente di pressione sarà in ogni tempo lo stesso per tutte le profondità, sotto un particolare punto della superficie del lago. Le accelerazioni saranno pertanto le stesse ad ogni profondità; in un istante determinato, saranno le stesse, sotto un particolare punto della superficie del lago, anche le correnti. Misure di corrente eseguite al centro del lago, daranno quindi un diagramma, del tipo riportato in fig. (14).

$\zeta$ e $u$ vanno considerate funzioni di $x, t$ tali da soddisfare alle condizioni $u=0$ per $x=o, x=l$, e di dare oscillazioni armoni-
Sostituendo [13] nella [11] si ha

$$
\frac{\partial \zeta}{\partial t}=-\pi \tilde{u} \frac{\pi}{l} \cos \frac{\pi x}{i} \sin \frac{2 \pi}{T_{o}}-t .
$$

Integrando si ha

$$
\zeta=\frac{T_{o} h}{2 l} C \cos \frac{\pi x}{l} \cos \frac{2 \pi}{T_{o}} t,
$$

senza costante additiva, il movimento essendo armonico nel tempo.

Per la [13] e la [14], la [12] diviene

$$
\begin{aligned}
& C \frac{2 \pi}{T_{o}} \sin \frac{\pi x}{l} \cos \frac{2 \pi}{T_{o}} t= \\
= & \frac{\pi T_{o}}{2 l^{2}} g h C \sin \frac{\pi x}{l} \cos \frac{2 \pi}{T_{J}} \stackrel{t}{,}
\end{aligned}
$$

da cui

$$
\frac{2}{T_{o}}=\frac{T_{c} g h}{2 l^{2}},
$$

e quindi

$$
T_{o}=\frac{2 l}{\sqrt{g h}},
$$


ben nota sotto il nome di formula di Merian. La [14] si trova spesso sotto la forma

$$
\zeta=H \cos \frac{\pi x}{l} \cos \frac{2 \pi}{\bar{I}_{o}} t,
$$

per cui

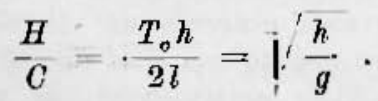

Il trascurare l'accelerazione verticale $\frac{\partial^{2} \zeta}{\partial t^{2}}$ implica sia piccolo il rapporto $\frac{h}{l}$; il che generalmente si verifica. all'energia costante totale dell'oscillazione, cioè $E$.

Poichè l'energia cinetica è proporzionale al quadrato della velocità delle correnti, segue che al tempo $t$ l'energia cinetica ha l'espressione

$$
E_{c}=E \sin ^{2} \frac{2 \pi}{T_{s}} t
$$

mentre l'energia potenziale è data da

$$
E_{p}=E-E \sin ^{2} \frac{2 \pi}{T_{o}} t=E \cos ^{2} \frac{2 \pi}{T_{o}} t
$$

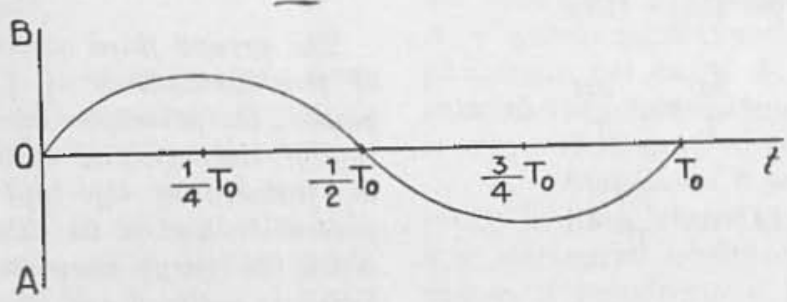

Fig. 14

Quanto più la forma del bacino di un lago si scosta da quella di un rettangolo, tanto più la formula di Merian dà per $T_{o}$ valori discosti da quelli effettivi.

Per il lago di Bracciano si ha $l=\mathrm{km} \mathrm{8,}$ $h=\mathrm{m} \mathrm{86,1}$

$$
T_{s}=9^{\mathrm{m}}, 15 \text {. }
$$

Il periodo calcolato con i metodi più approssimati - coincidente con il medio periodo osservato - ̀̀ $8^{\mathrm{m}}, 3$. Quindi

$$
\frac{H}{C}=2,97 \mathrm{sec} \text {. }
$$

Per $H-4 \mathrm{~cm}$ (valore massimo delle ampiezze dell'unnodale del lago di Bracciano), si ha

$$
C=1,36 \mathrm{~cm} / \mathrm{sec} .
$$

Con riferimento alle figg. 13 e 14, nell'uninodale ai tempi $t-o, t=\frac{{ }_{2}}{2} T_{\mathrm{o}}$ l'energia è tutta potenziale, mentre ai tempi $t=\frac{1}{-} T_{o}$ e $t=\frac{3}{-} T_{o}$ l'energia è tutta cinetica. Per il principio della conservazione dell'energia, ognuna di queste due energie è uguale
Per bacini allungati, di sezione variabile, valgono le [11], [12]. Dalla [12] si ha, moltiplicando per $S u$ ed integrando fra gli estremi $x=x_{1}$, e $x=x_{2}$ del lago:

$$
\int_{\dot{x}_{1}}^{2} S u \frac{\partial u}{u t} d x=-g \int_{x_{1}}^{x_{2}} S u \frac{\partial \zeta}{\partial x} d x .
$$

Da cui, portando fuori dal segno d'integrale a primo membro il simbolo di derivazione rispetto al tempo e integrando il secondo membro per parti

$$
\begin{gathered}
\frac{x_{2}}{\partial t} \int^{x_{2}} S u^{2} d x= \\
=-g|S u \zeta|^{x_{2}}+a \mid S u \cdot \frac{\partial x}{\partial x}(A u) \zeta d x
\end{gathered}
$$

Ma agli estremi del lago è $S u=0$, per eui — ricordando la [11] —

$$
\frac{\partial}{\partial t} \frac{2}{2} \int^{2} S u^{2} \partial x=g \mid b \zeta \frac{\partial \zeta}{\partial t} d x,
$$


da cui, integrando,

$\frac{1}{2} \int_{x_{1}}^{x_{2}} S u^{2} d x+\frac{1}{2} g \int_{x_{1}}^{x_{2}} b \zeta^{2} d x=$ costante.

I] primo membro, moltiplicato per la densità $\varrho$ dell'acqua, dà la somma delle energie cinetica e potenziale; pertanto, la conservazione dell'energia consegue - in questo caso - deducendo la [19] dalle [11], [12], cioè dalle equazioni della continuità e del moto.

Nel caso speciale di un bacino rettangolare (essendo $S=b h$ e per [13] e [15])

$$
\begin{gathered}
E_{c}=\frac{1}{2} \varrho \int_{o}^{l} b h C^{2} \sin ^{2} \frac{\pi x}{l} \sin ^{2} \frac{2 \pi}{T_{o}} t d x= \\
=\frac{1}{4} \varrho b h l C^{2} \sin ^{2} \frac{2 \pi}{\bar{T}_{o}} t, \\
E_{p}=\frac{1}{2} g \varrho \int_{o}^{l} b H^{2} \cos ^{2} \frac{\pi x}{l} \cos ^{2} \frac{2 \pi}{T_{o}^{\prime}} t d x= \\
=\frac{1}{4} \cdot g \varrho b l H^{2} \cos ^{2} \frac{2 \pi}{T_{o}} t .
\end{gathered}
$$

Tenendo conto della $[16]$, si vede che restano verificate le [17], [18].

Nel caso del lago di Bracciano, facendo $\varrho=1 \mathrm{gr} / \mathrm{cm}^{3}, l=8 \mathrm{~km} ; b=5 \mathrm{~km}, h=$

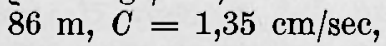

$$
\begin{aligned}
E & =1,6 \cdot 10^{15} \mathrm{ergs} \\
& =44 \mathrm{kwh} .
\end{aligned}
$$

Tale è il valore medio dell'energia associata alle oscillazioni libere uninodali del lago di Bracciano.

\section{RIASSUNTO}

In questo terzo contributo allo studio delle oscillazioni libere del lago di Bracciano, si espongono $i$ principi teorici sui quali $\dot{e}$ basato il funzionamento dei limnografi $e$ si discutono le caratteristiche del tipo di limnografo, attualmente in funzione sui laghi dell'Italia centrale. Si accenna quindi all'energia associata ad un'oscillazione libera e al modo di determinarla. Le sesse del lago di Bracciano sono generalmente di piccola ampiezza. Per quanto concerne l'oscillazione libera uninodale asimmetrica, si prova che l'energia media associata a questo tipo di sessa è dell'ordine di $44 \mathrm{kWh}$.

\section{$A B S T R A C T$}

The present third contribution to the study of free oscillations of Lake Bracciano expounds the principles on which the functioning of limnographs is based and describes the features of the type of limnograph at present in use on the lakes of Central Italy. Next, the energy associated with a free oscillation is outlined and the way of determining it is indicated. The seiches of Lake Bracciano are generally of small amplitude. As regards the free uninodale assymmetric oscillation, it is demonstrated that the average energy associated with this type of seiche reaches about $44 \mathrm{kWh}$.

\section{BIBLIOGRAFIA}

(1) CaLor, P., Sui possibili modi di oscillazione libera del lago di Bracciano. "Ann. di Geofisica ", III, 1, (1950).

$\left({ }^{2}\right)$ - \& Peronaci, F., Oscillazioni libere e forzate del lago di Bracciano. "Arch. di Oceanogr. e Limn.") (in corso di stampa).

(3) Peronaci, F., Limnograjo a pressione. "Anu. di Geofisica ", III (1950).

(') OsBorne Reynolds, On the Motion of Water and on the Law of Resistance in Parallel Channels. "Phil. Trans. Roy. Soc. Lond" (1883).

$\left({ }^{5}\right)$ Perucca, E., Fisica generale e sperimentale. Vol. I, pag. 389, prima ediz. (1932).

(6) Proudina, J., Dynamical Oceanography. London, 1953. 